\title{
Exact Calculation of the Linear Term in the Density Expansion of the Dynamic Structure Factor of a Dilute Gas
}

\author{
Kunimasa Miyazaki* and I. M. de Schepper \\ IRI, Delft University of Technology, 2629 JB Delft, The Netherlands \\ (Received 15 June 2001; published 5 November 2001)
}

\begin{abstract}
We evaluate the linear term in the density expansion of the dynamic structure factor for a classical gas using a generalized Enskog theory developed by one of the authors, which enables us to describe the dynamics at small time and length scales where the use of the Boltzmann equation is severely limited. Agreement of the theory with experiment and simulation is very good. We find that the linear term is very sensitive to the shape of the potential so that the dynamic structure factor can serve as a good probe to determine the intermolecular potentials of dilute gases.
\end{abstract}

DOI: $10.1103 /$ PhysRevLett.87.214502

The static properties of moderately dense gases can be expressed systematically in power series expansions in the number density $n$, where the leading term $n=0$ refers to the ideal gas and the higher terms involve the intermolecular potentials between the particles. The virial expansion of the equation of state is the best known example [1]. Also, neutron diffraction has been applied on rare gases [2-4] to measure the static structure factor $S(k)$ as a function of wave vector $k$ and $n$, where $S(k)=1$ for $n=0$ refers to the ideal gas. From the coefficients in the density expansion of $S(k)$, one obtains directly the microscopic interactions between the particles in an exact manner, in particular, the pair potential $\phi(r)$ as a function of interparticle distance $r$. The dynamic properties of moderately dense gases (such as transport coefficients) have been treated in a similar manner. Here, to describe the first order corrections to the ideal gas limit, one needs the Boltzmann equation for dilute gases. This equation, defined for any intermolecular potential, $\phi(r)$, is exact for $n \rightarrow 0$ as long as the observed length scale $r \gg \sigma$ with $\sigma$ a typical diameter of the particles and time scale $t \gg$ $\tau_{\text {int }}=\sigma / v_{\text {th }}$, where $\tau_{\text {int }}$ is a typical interaction time, $v_{\text {th }}=4 / \sqrt{2 \pi \beta m}$ is the thermal velocity of the particles with mass $m$ at temperature $T$, and $\beta=1 / k_{\mathrm{B}} T$. Thus $\tau_{\text {int }}$ is the time a particle needs to travel the distance $\sigma$ with thermal velocity $v_{\text {th }}$. These two limitations are due to the fact that Boltzmann assumed pointlike particles colliding instantaneously which is correct when $r \gg \sigma$ and $t \gg \tau_{\text {int }}$. Recently, Verkerk et al. have conducted inelastic neutron scattering experiments and observed the linear term in the density expansion of the dynamic structure factor, $S(k, \omega)$, for dilute argon for the first time [5]. The results as a function of $k$ and frequency $\omega$ were compared with the theoretical prediction obtained using the Boltzmann equation, which reads

$$
\frac{S(k, \omega)}{S(k)}=S^{(0)}(k, \omega)+n S^{(1)}(k, \omega)+\mathcal{O}\left(n^{2}\right),
$$

where $S(k)=\int_{-\infty}^{\infty} d \omega S(k, \omega)$ is the static structure factor tending to 1 for $n=0$. The leading term describes the ideal gas behavior, i.e.,
PACS numbers: $51 \cdot 10 .+\mathrm{y}$

$$
S^{(0)}(k, \omega)=\frac{2}{\pi k v_{\mathrm{th}}} e^{-4 \omega^{* 2} / \pi},
$$

with the dimensionless reduced frequency $\omega^{*}$ given by $\omega^{*}=\omega / k v_{\text {th }}$. The first order correction term has been written as [6]

$$
S^{(1)}(k, \omega)=\frac{2}{\pi k v_{\mathrm{th}}} \frac{s_{11}(k, \omega)}{k \ln },
$$

with $l=1 / \sqrt{2} \pi n \sigma^{2}$ the mean free path between collisions so that $\ln$ is independent of $n$. It is a direct consequence of the Boltzmann equation that $s_{11}(k, \omega)=$ $s_{11}\left(\omega^{*}\right)$ depends only on $\omega^{*}$ (not on $k$ ), where the shape of $s_{11}\left(\omega^{*}\right)$ depends on the intermolecular potential $\phi(r)$. In Ref. [5], it was found that the experimental result for $S(k, \omega)$ agrees qualitatively well with the Boltzmann result of Eq. (3) for hard spheres at small wave vectors $k$ but, as $k$ increases, the discrepancies are pronounced, especially at small frequencies. Most notably, these experiments showed that $s_{11}(k, \omega)$ is not a single function of $\omega^{*}$ but also sensitively depends on $k$, exhibiting the limitation of validity of the Boltzmann equation itself even when the system is ideally dilute. Recent experiments [7] at even lower densities $n$ confirmed these observations. Bafile et al. $[8,9]$ implemented a molecular dynamics simulation for a Lennard-Jones gas with parameters corresponding to the experiments, again showing that $s_{11}(k, \omega)$ not only depends on $\omega^{*}$ but also on $k$. In addition, we have shown previously [10] that, using the Boltzmann equation, the function $s_{11}\left(\omega^{*}\right)$ for hard spheres is not significantly different from that calculated for Lennard-Jones potentials. Hence, to understand the experiments [5] and the molecular dynamics simulation [8,9], one needs a theory which extends the Boltzmann approach beyond its range of validity, i.e., to small times (large frequencies) $t \lesssim \tau_{\text {int }}$ and small lengths (large wave vectors) $r \lesssim \sigma$. One can use the revised Enskog theory $[6,11]$ to explore the dynamics of the arbitrarily large wave vectors but this method is limited to hard-sphere fluids.

Recently, Miyazaki et al. have developed a novel method to calculate correlation functions of dilute and 
semidilute gases with arbitrary potentials [12,13]. They derived a frequency and wave-vector-dependent "Enskogtype" collision operator which enables us to explore arbitrarily short length and short time regimes.

In this Letter, we apply this generalized "Enskog theory" and calculate exactly the linear term in the density expansion of $S(k, \omega)$ for the first time and explain the experimental and simulation results. We show that the linear term is dependent on the wave vector and really sensitive to the potential shape.

Here, we consider only a classical gas interacting with the (12-6) Lennard-Jones potential defined by $\phi(r)=$ $4 \epsilon\left\{(\sigma / r)^{2}-(\sigma / r)^{6}\right\}$, where $\epsilon$ is the depth of potential, and $\sigma$ is the distance at which $\phi(r)=0$. The dynamic structure factor is defined as the Fourier transformation of the correlation function of the total density and written as

$$
S(k, \omega)=\frac{1}{2 \pi} \int_{-\infty}^{\infty} d t e^{i \omega t} \frac{1}{N} \sum_{i, j=1}^{N}\left\langle e^{i \mathbf{k} \cdot\left\{\mathbf{R}_{i}(0)-\mathbf{R}_{j}(t)\right\}}\right\rangle,
$$

where $N$ is the total number of the atoms, $\mathbf{R}_{i}(t)$ is the position of the $i$ th atom at a time $t$, and $\langle\cdots\rangle$ is the average over an equilibrium ensemble at temperature $T$. We can expand this in terms of the density, leading to Eqs. (1)-(3). Using the Boltzmann equation and the corresponding Boltzmann operator, $\Lambda_{B}, s_{11}(k, \omega)$ can be written as $[6,10]$

$$
\begin{aligned}
s_{11}(k, \omega)= & \sqrt{\frac{1}{\pi^{3} m \beta}}\left(\frac{k}{\sigma}\right)^{2} \\
& \times \operatorname{Re}\left\langle\frac{1}{i \omega+i \mathbf{k} \cdot \mathbf{v}} \Lambda_{B} \frac{1}{i \omega+i \mathbf{k} \cdot \mathbf{v}}\right\rangle,
\end{aligned}
$$

where $\mathbf{v}$ is the actual velocity of an atom. It was shown that $s_{11}(k, \omega)$ is a function of $\omega^{*}$ only and independent of $k$. If $k^{-1}$ is the order of interaction length $\sigma$ or $\omega^{-1}$ is comparable with the mean interaction time $\tau_{\text {int }}$, one cannot use $\Lambda_{B}$ anymore and it should be replaced by a wave-vector- and frequency-dependent alternative, a so-called generalized Enskog operator, $\Lambda_{E \mathbf{k}}(\omega)$. According to Refs. [12,13], it is written, in the low density limit, as $\Lambda_{E \mathbf{k}}(\omega)=M_{\mathbf{k}}+\Gamma_{\mathbf{k}}(\omega) . M_{\mathbf{k}}$ is the mean field operator defined, for an arbitrary function of velocity $A(\mathbf{v})$, by

$$
M_{\mathbf{k}} A(\mathbf{v})=\frac{h(k)}{2} \int d \mathbf{v}^{\prime} f_{0}\left(v^{\prime}\right) i \mathbf{k} \cdot\left(\mathbf{v}+\mathbf{v}^{\prime}\right) A\left(\mathbf{v}^{\prime}\right),
$$

where $h(k)$ is the pair correlation function given by the Fourier transformation of $e^{-\beta \phi(r)}-1$, and $f_{0}(v)$ is the

Maxwellian distribution function. $\Gamma_{\mathbf{k}}(\omega)$ is the binary collision operator given, in real time representation, by

$$
\begin{aligned}
\Gamma_{\mathbf{k}}(t) A(\mathbf{v})= & \frac{1}{f_{0}(v)} \int d \mathbf{r} \int d \mathbf{v}^{\prime} e^{-i \mathbf{k} \cdot \mathbf{r} / 2} i L_{I} e^{-i \mathbf{k} \cdot\left(\mathbf{v}+\mathbf{v}^{\prime}\right) t / 2} \\
& \times e^{-\beta \phi(r)} f_{0}(v) f_{0}\left(v^{\prime}\right) e^{-i L t} i L_{I}\left\{e^{i \mathbf{k} \cdot \mathbf{r} / 2} A(\mathbf{v})\right. \\
& \left.+e^{-i \mathbf{k} \cdot \mathbf{r} / 2} A\left(\mathbf{v}^{\prime}\right)\right\},
\end{aligned}
$$

where $i L$ is the Liouville operator for a pair of atoms which have initial velocities, $\mathbf{v}$ and $\mathbf{v}^{\prime}$, and are separated by a distance $\mathbf{r}$. $i L_{I}$ is the interaction term of $i L$. The time evolution operator $e^{-i L t}$ acts on all terms on the right-hand side of it. Thus, one needs the trajectories, $\mathbf{v}(t), \mathbf{v}^{\prime}(t)$, and $\mathbf{r}(t)$, of a pair of colliding atoms at arbitrary time $t$. They are given by the solution of the equation of motion for two particles. In polar coordinates, it is given by

$$
\begin{gathered}
t=\int^{r(t)} d r^{\prime} f\left(r^{\prime}\right) \quad \text { and } \\
\theta(t)=2 v r \sin \theta \int^{r(t)} d r^{\prime} \frac{f\left(r^{\prime}\right)}{r^{\prime 2}},
\end{gathered}
$$

where $f(r)=1 / \sqrt{4\{E-\phi(r)\} / m-4 v^{2} \sin ^{2} \theta}$, and $E$ is the total energy for a pair of atoms. The constants of integration can be chosen such that the solutions satisfy the initial conditions, $\mathbf{r}(t=0)=\mathbf{r}$, etc. For the hard-sphere case, Eqs. (6) and (7) reduce to those given in Refs. [6,11]. In the small $k$ and $\omega$ limit, they become the familiar Boltzmann operator $[10,13,14]$. By operating $\Lambda_{E \mathbf{k}}(\omega)$ on $A(\mathbf{v})=(i \omega+i \mathbf{k} \cdot \mathbf{v})^{-1}$, we can evaluate $s_{11}(k, \omega)$. The final expression is written as $s_{11}(k, \omega)=$ $s_{11}^{(a)}(k, \omega)+s_{11}^{(b)}(k, \omega)$. The first term is the mean field contribution given, in units where $m=\sigma=k_{\mathrm{B}}=1$, by

$$
\begin{aligned}
s_{11}^{(a)}(\omega, k)= & \frac{k h(k)}{\sqrt{2} \pi} e^{-4 \omega^{* 2} / \pi} \\
& \times\left(1-\frac{8 \omega^{*}}{\sqrt{\pi}} e^{-4 \omega^{* 2} / \pi} \int_{0}^{\left(2 \omega^{*} / \sqrt{\pi}\right)} d x e^{x^{2}}\right),
\end{aligned}
$$

and the second term is the binary collision contribution given by

$$
s_{11}^{(b)}(k, \omega)=\frac{1}{\pi} \int_{0}^{\infty} d t F(k, t) \cos \omega t,
$$

where

$$
\begin{aligned}
F(k, t)= & -\frac{2 k^{2}}{\pi^{3} T} e^{-T k^{2} t^{2} / 4} \int_{0}^{t} d t_{1} \int d \mathbf{v} \int d \mathbf{r} e^{-\left[v^{2}+\phi(r)\right] / T} \mathbf{k} \cdot \nabla \phi(r) \mathbf{k} \cdot \nabla \phi\left(r_{1}\right) t_{1}^{3} \\
& \times\left[\psi\left(\mathbf{k} \cdot\left\{\delta \mathbf{r}_{1}+\mathbf{v} t_{1}\right\}, \mathbf{k} \cdot\left\{\delta \mathbf{r}_{1}+\mathbf{v}_{1} t_{1}\right\}\right)+\psi\left(\mathbf{k} \cdot\left\{\mathbf{r}+\delta \mathbf{r}_{1}-\mathbf{v} t_{1}\right\}, \mathbf{k} \cdot\left\{\mathbf{r}+\delta \mathbf{r}_{1}+\mathbf{v}_{1} t_{1}\right\}\right)\right],
\end{aligned}
$$

where $\psi(x, y)=\partial^{2} / \partial x \partial y(\sin x-\sin y) /(x-y), \delta \mathbf{r}_{1} \equiv$ $\left(\mathbf{r}_{1}-\mathbf{r}\right) / 2$, and $\mathbf{r}_{1}, \mathbf{v}_{1}$ denote the position and velocity at time $t-t_{1}$, respectively. Note that neither $s_{11}^{(a)}(k, \omega)$ nor $s_{11}^{(b)}(k, \omega)$ is a function of $\omega^{*}$ only. We have evaluated the multidimensional integration of the above expres- sion numerically using the Gaussian quadrature routine. After evaluating $F(k, t)$, the fast Fourier transform (FFT) routine was used to transfer to $s_{11}^{(b)}(k, \omega)$. Lennard-Jones parameters used in the calculations are those for argon; 
$\sigma=0.34 \mathrm{~nm}$ and $\epsilon / k_{\mathrm{B}}=120 \mathrm{~K}$. In Fig. 1, we show the results for four wave vectors along with the result of the experiment [5] and the molecular dynamics simulation [8,9]. All results are for temperature $T=300 \mathrm{~K}$.

Our theory agrees well with experiment, at least semiquantitatively. The agreement with the simulation results is very good for all wave vectors. Considering the difficulties in experiments and simulation to separate $s_{11}(k, \omega)$ from the ideal gas contribution, the agreement is regarded as satisfactory. One sees that $s_{11}(k, \omega)$ is really sensitive to the wave vector. Both the mean field term $s_{11}^{(a)}(k, \omega)$ and the binary collision term $s_{11}^{(b)}(k, \omega)$ are found to be sensitive to the wave vector. For small wave vectors, the results are qualitatively similar to those calculated from the Boltzmann equation. But, as the wave vector increases, the difference with the Boltzmann results is pronounced; the amplitudes shrink and the values at small $\omega^{*}$ become positive for large $k$, a feature seen in both experiment and simulation.

One might conjecture that this behavior at large $k$ is the crossover of collective to single atom dynamics. It is known that, in the large wave vector limit, the dynamics of collective quantities becomes indistinguishable from that of a single atom. The linear term in the density

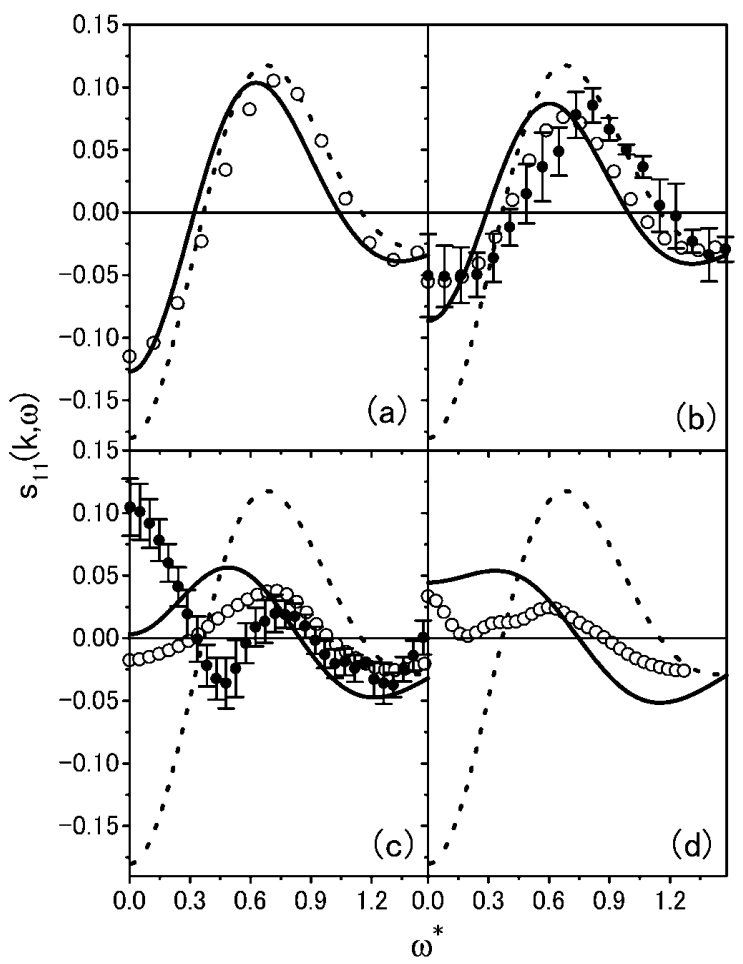

FIG. 1. $s_{11}(k, \omega)$ for argon for several wave vectors: (a) $k=$ $0.5 \mathrm{~nm}^{-1}$, (b) $k=0.71 \mathrm{~nm}^{-1}$, (c) $k=1.22 \mathrm{~nm}^{-1}$, and (d) $k=$ $1.5 \mathrm{~nm}^{-1}$. Solid lines are from the present theory. The dotted line is the result from Boltzmann theory for the hard sphere according to Kamgar-Parsi [6]. The filled circles in (b) and (c) are experimental results by Verkerk [5] for $k=0.7$ and $1.2 \mathrm{~nm}^{-1}$, respectively. Empty circles are molecular dynamics simulation results by Bafile et al. $[8,9]$. expansion of the dynamic structure factor for a single atom, $s_{11}^{s}(k, \omega)$, was calculated by Montfrooij et al. [15] for a hard-sphere gas using the Lorentz-Boltzmann operator, the single atom counterpart of the Boltzmann operator, $\Lambda_{B}$. For hard spheres, $s_{11}^{s}(k, \omega)$ exhibits nearly out-of-phase behavior from $s_{11}(k, \omega)$ as shown by the dotted lines in Fig. 1. However, what is observed for large $k$ is not this crossover. A generalized Enskog operator for single atom motion is given by a similar operator as $\Lambda_{E \mathbf{k}}(\omega)$. The only difference is the absence of the mean field contribution [Eq. (6)] and the second term in parentheses of $\Gamma_{\mathbf{k}}(t)$ in Eq. (7). The differences between the two operators disappear as $k$ increases because of the phase randomization of the $e^{i \mathbf{k} \cdot \mathbf{r}}$ terms in $h(k)$ and in Eq. (7). Combining this with the fact that the major contribution of the integral over $r$ in Eq. (7) originates from values of the integrand around $r \simeq \sigma$, we estimate that the crossover to single atom motion occurs at $k \sigma \gg 1$. The highest wave vector in Fig. 1, however, corresponds to $k \sigma=0.51$, which is far too small to see any crossover. We have checked this by calculating both $s_{11}(k, \omega)$ and $s_{11}^{s}(k, \omega)$ for larger $k$. We found that the apparent hike of the line from negative to positive values in Fig. 1 is a mere oscillation present at small $k \sigma$. We confirmed that the crossover occurs only when $k \geq 15 \mathrm{~nm}^{-1}$; wave vectors far beyond the limit the experiment can explore. For such large wave vectors, however, $1 / k$ becomes comparable with the thermal de Broglie wavelength and, therefore, a quantum mechanical analysis will be required [16].

Now that we have the tool in hand to calculate $s_{11}(k, \omega)$ for arbitrary potentials, wave vectors, and frequencies exactly, we can go back to the original question posed by Verkerk et al.: Is $s_{11}(k, \omega)$ really sensitive to the potential shape? In order to answer this question, we calculated $s_{11}(k, \omega)$ for both Lennard-Jones and hard-sphere potentials. The effective hard-sphere diameter corresponding to the Lennard-Jones gas, $\sigma_{\text {eff }}$, is determined as a distance where the Lennard-Jones atom hits the repulsive part of the potential, i.e., $\phi\left(\sigma_{\text {eff }}\right)=k_{\mathrm{B}} T$. The result is shown in Fig. 2 for the lowest and highest wave vectors considered in the simulation.

For $k=0.5 \mathrm{~nm}^{-1}$, the difference between the two potentials is conceivable but not as big as we have seen in the previous paper [10]. As the wave vector increases, each line starts to deviate from that at $k=0.5 \mathrm{~nm}^{-1}$ but in the opposite direction and, at $k=1.5 \mathrm{~nm}^{-1}$, the overall behavior is completely different. We have also calculated $s_{11}(k, \omega)$ for larger $k$ and found that, in the large- $k$ limit, both Lennard-Jones and hard-sphere results approach $s_{11}^{s}(k, \omega)$ which is insensitive to the potential shape. But their behavior in the crossover region around $k \approx 15 \mathrm{~nm}^{-1}$ is completely different.

In conclusion, we have confirmed that the linear term of the density expansion of the dynamic structure factor is indeed sensitive to the shape of the intermolecular potential. Therefore, as Verkerk et al. have expected, probing the 


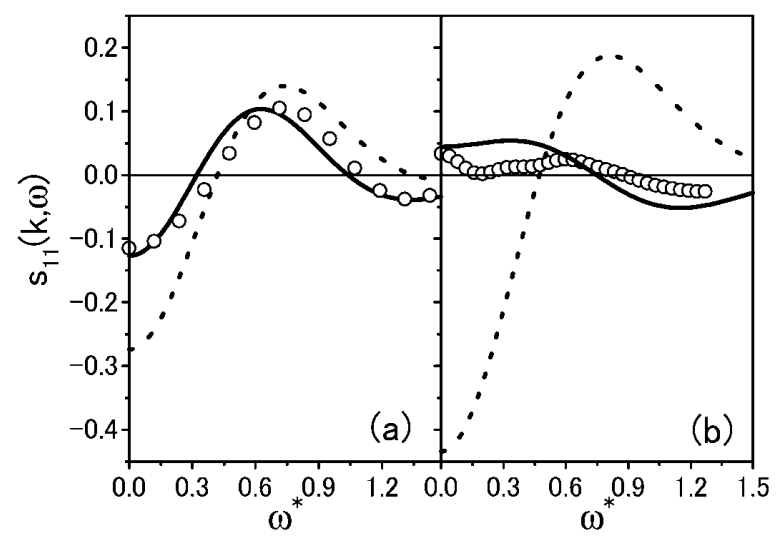

FIG. 2. Comparison between the Lennard-Jones potential and hard-sphere potential for (a) $k=0.5 \mathrm{~nm}^{-1}$ and (b) $k=$ $1.5 \mathrm{~nm}^{-1}$. The solid lines are for the Lennard-Jones and the dotted lines are for the hard sphere. The molecular dynamics simulation results by Bafile et al. are shown by empty circles.

dynamic structure factor of dilute gases can be as direct and powerful a way to investigate the intermolecular potential as the static correlation such as $S(k)$. This sensitivity might explain rather large discrepancies between the experimental results from theory and simulation in Fig. 1(c). The discrepancy might be attributed to the difference of the real potential for argon from the Lennard-Jones potential.

We are grateful to P. Verkerk for providing us with his experimental data and fruitful discussions. We thank Professor Biman Bagchi for encouragement and enlightening discussion. The work is supported in part by grants from Japan Society for the Promotion of Science (JSPS).
*Electronic address: k.miyazaki@iri.tudelft.nl

[1] D. A. McQuarrie, Statistical Mechanics (HarperCollins, New York, 1973).

[2] A. Teitsma and P. A. Egelstaff, Phys. Rev. A 21, 367 (1980).

[3] F. Barocchi, M. Zoppi, and P. A. Egelstaff, Phys. Rev. A 31, 2732 (1985).

[4] H. Fredrikze, J. B. van Tricht, A. A. van Well, R. Magli, P. Chieux, and F. Barocchi, Phys. Rev. Lett. 62, 2612 (1989).

[5] P. Verkerk, U. Bafile, F. Barocchi, L.A. de Graaf, J.-B. Suck, and H. Mutka, Phys. Rev. Lett. 67, 1262 (1991).

[6] B. Kamgar-Parsi, E. G. D. Cohen, and I. M. de Schepper, Phys. Rev. A 35, 4781 (1987).

[7] B. Mos, Ph.D. thesis, Delft University of Technology, 2000.

[8] U. Bafile, F. Barocchi, and M. Neumann, J. Mol. Struct. 296, 221 (1993).

[9] U. Bafile, F. Barocchi, and M. Neumann, Phys. Rev. E 51, 3756 (1995).

[10] K. Miyazaki and I. M. de Schepper, Phys. Rev. E 63, 060201 (2001).

[11] I. M. de Schepper and E. G. D. Cohen, J. Stat. Phys. 27, 223 (1982).

[12] K. Miyazaki, G. Srinivas, and B. Bagchi, J. Chem. Phys. 114, 6276 (2001).

[13] K. Miyazaki, I. M. de Schepper, and B. Bagchi, Physica (Amsterdam) 298A, 101 (2001).

[14] P. Résibois and M. de Leener, Classical Kinetic Theory of Fluids (Wiley, New York, 1977).

[15] W. Montfrooij, P. Verkerk, and I. M. de Schepper, Phys. Rev. A 33, 540 (1986).

[16] M. Moraldi, M. Celli, and F. Barocchi, Phys. Rev. A 46, 7561 (1992). 